\title{
The Health Evaluation of Wetland Ecosystem in the Yangtze River Economic Zone Based on Remote Sensing_-A Case Study of Yunnan Wetland
}

\author{
Haonan Wang ${ }^{1,2}$, Fan $\mathrm{Yu}^{1,2, *}$, and Jun $\mathrm{Li}^{3}$ \\ ${ }^{1}$ Lanzhou Institute of drought meteorology, 730020 Lanzhou, China \\ ${ }^{2}$ Beijing University of Civil Engineering and Architecture, 102616 Beijing, China \\ ${ }^{3}$ State Geospatial Information Center, 100070 Beijing, China
}

\begin{abstract}
In order to provide a scientific basis for the development of the Yunnan wetland ecosystem, we construct a wetland ecosystem health evaluation system with 15 evaluation indexes selected from three aspects including pressure, status and response based on PSR mathematical model. Analytic Hierarchy Process(AHP) was used to determine the weight of indicators and the Yunnan wetland ecosystem was divided into "the five health status" of "health, sub-health, fragility, illness, and scurviness", which are used to analyze and evaluate the health status of it through a comprehensive evaluation index. The results show that the comprehensive evaluation index of ecosystem health status of Yunnan wetland is 0.5524 in 2017, locating in the grade of "fragility" and is close to the status of "sub-healthy"; Among the 3 levels of pressure, state, and response, the pressure and state are locating in the grade of "fragility" and the response is close to the status of "health". Among the 15 evaluation indexes, the rate of change of wetland area, the area of water body, the hydrological regulation and the wetland management level are the most important factors affecting the ecosystem health of Yunnan wetland.
\end{abstract}

\section{Introduction}

Wetland is a unique ecosystem formed by the interaction of land and water on the earth, which is an important living environment and one of the most biologically diverse ecological landscapes in nature. And it has an irreplaceable role in resisting floods, regulating runoff, improving climate, controlling pollution, beautifying the environment and maintaining regional ecological balance. Wetland is also known as "the kidney of the earth". Therefore, in the World Natural Protection Program, wetland is listed as the three major global ecosystems together with forests and oceans1.

In the field of wetland research, wetland ecosystem health research is a new direction2. In recent years, domestic scholars have done many related studies on the health of China's regional wetland ecosystems. Based on wetland environmental health evaluation methods, and combined with the specific characteristics of Dongting Lake wetland, Mao Xiaoqian took Dongting Lake wetland as the research area, analyzing the type and quantity characteristics of biological elements of it for 5 consecutive years3. Based on the PSR model, and adopted the Fuzzy-AHP method, according to the comprehensive evaluation index, Wang Fuqiang et al. took the Diaokou River Wetland as an example, analyzing the wetland ecosystem health status in different periods4. Xu Ye et al. took the Xiong'an urban

\footnotetext{
* Corresponding author: yufan@bucea.edu.cn
}

wetland as the example and used Fragstats software to analyze the landscape pattern of it, and the PSR model was used to construct a wetland ecosystem health evaluation index system in Xiong'an City, analyzing the wetland ecosystem health status5. Above all, the health evaluation system established by most domestic scholars is limited to the study of a single wetland6. As the relationship between humans and the environment is getting closer and closer, local evaluation of the health of an ecosystem cannot objectively and accurately analyze the health of wetlands. Only by linking economy, culture and politics, can we provide new ideas for the future development trend of wetlands.

Meanwhile, it is difficult to obtain on-site data on wetland ecosystems, so remote sensing technology has become a new method to investigate wetland resources and has detected its dynamic changes2. Domestic scholars have done a lot of research on this basis. Ma Chi introduced various analysis methods such as Markov transition matrix, center of gravity migration, landscape pattern and so on to explore the characteristics of the dynamic changes in the time and space of wetlands in Northeast China7. Zhu Chunjiao et al. analyzed the characteristics and laws of wetland evolution, and used Markov and CLUE-S models to predict wetland changes in Pudong8. Suo Anning et al. used applied environmental economics, resource economics and other methods to evaluate the service function value of the Liaohe Delta wetland ecosystem9. Wang Lichun et al. 
used RSEI index to monitor and evaluate the ecological environment of Manas Lake wetland10.

Based on the pressure-state-response framework model, this paper takes Yunnan wetlands as the example, using remote sensing images, and statistical data to establish a wetland ecosystem health evaluation system, and uses the Analytic Hierarchy Process and comprehensive evaluation method, combining with ecological health evaluation indicators and their grade standard to analyze the health changes of Yunnan wetland ecosystem. It provides a basis for accurate evaluation and scientific development of wetland ecosystem health, and at the same time provides decision support for the protection and management of wetland.

\section{Study area}

Yunnan Province is located on the border of southwest China, with geographic coordinates of $97^{\circ} 31^{\prime}-106^{\circ} 11^{\prime} \mathrm{E}$, $21^{\circ} 8^{\prime}-29^{\circ} 15^{\prime} \mathrm{N}$, and its total land area is $3.941 \times 10^{5} \mathrm{~km}^{2}$.

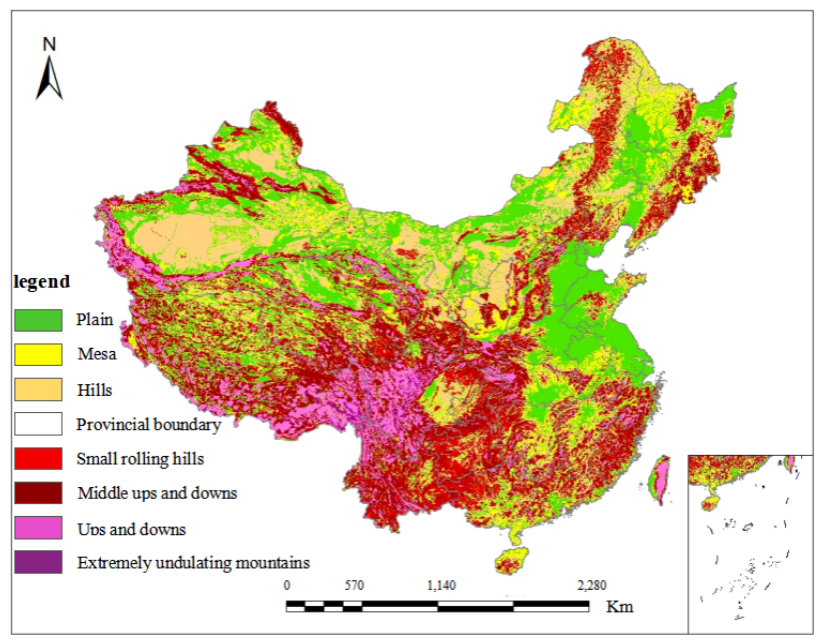

Fig. 1. Surface coverage map of Study area

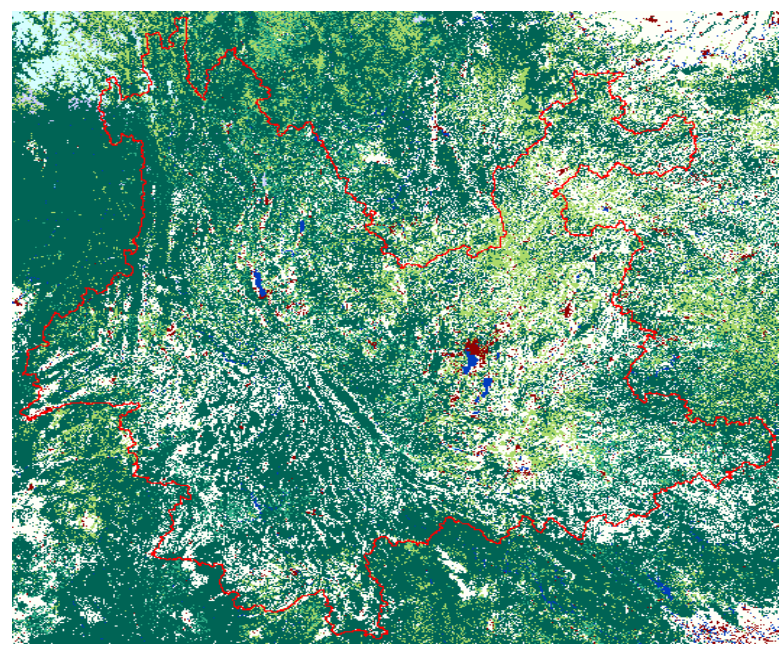

The spatial distribution characteristics are shown in Figure 1. The terrain is high in the northwest and it is low in the southeast, descending step by step from north to south. For every kilometer of horizontal straight line distance from north to south, the altitude decreases by an average of $6 \mathrm{~m}$. It belongs to the subtropical plateau monsoon climate, with remarkable three-dimensional climate characteristics, numerous types, small annual temperature differences, large daily temperature differences, distinct dry and wet seasons, and unusual vertical changes in temperature with the terrain. Yunnan wetland is an important part of China's wetland. It has the characteristics of wide distribution and many plateau freshwater lake wetlands. It is the current province with the largest number of freshwater lake wetlands in southwestern China. Its biodiversity occupies a very important position. It depends on wetlands for survival and reproduction. Wild animal and plant resources are extremely rich 11 .

radiometric calibration, atmospheric correction and

\section{Research methods and data sources}

\subsection{Data sources and preprocessing}

This study selected Landsat TM images on July 1st, in 2016, July 24th, in 2017, and August 16th, in 2018 as the remote sensing data source. The image quality is good, and the cloud cover is small, in order to avoid the influence caused by seasonal differences. The data source is preprocessed in ENVI 5.2 software, mainly for geometric correction (Figure 2). Among them, the spatial distribution data of 1:1 million landform types in China comes from the "Atlas of the People's Republic of China Geomorphology (1: 1 million)", and the other data sources, such as society, economy, and nature and so on are selected from the Yunnan Provincial Statistics Bureau, which is quantified. 


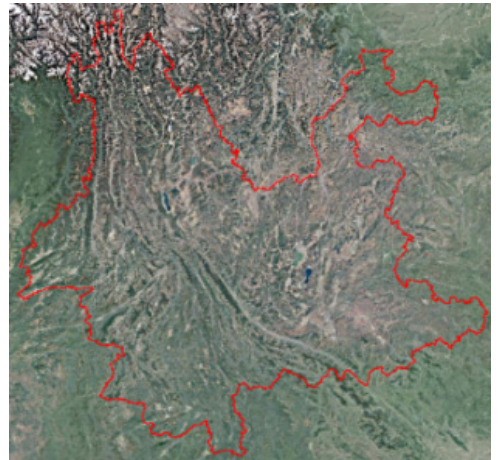

a.2016

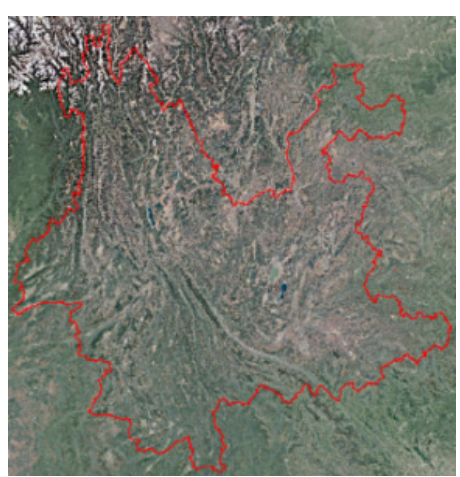

b. 2017

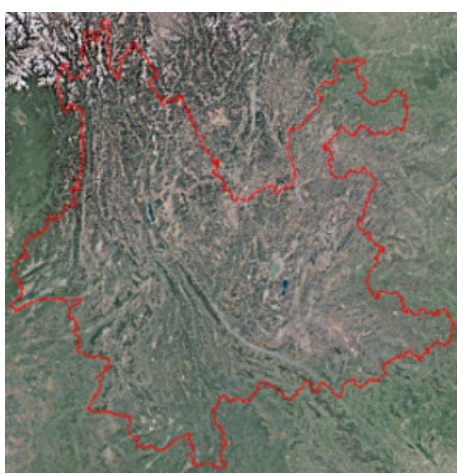

c. 2018

Fig. 2. Historical image of Yunnan wetland

\subsection{Research methods}

\subsubsection{PSR framework system}

The PSR model is a commonly used evaluation model for evaluating the health of ecosystems. It is based on pressure-state-response and combines the practical activities of human beings with human themselves. It is a macroscopic and microscopic point of view. In the evaluation of wetland ecological health, the criterion layer is selected from five aspects: economy, politics, culture, society and ecology. At the same time, the PSR model is adopted by many governments and research workers and is considered to be the most effective framework for evaluating environmental quality as shown in Figure 32.

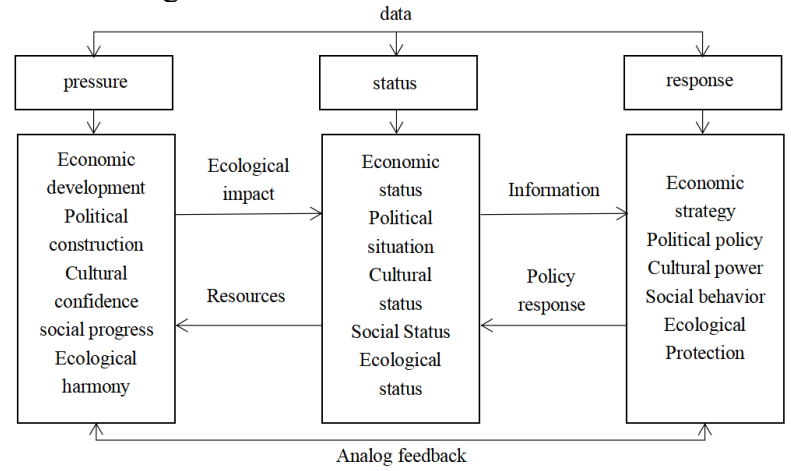

\subsubsection{The Analytic Hierarchy Process}

The analytic hierarchy process is a simple method to make decisions on some more complex and vague problems, and decompose the elements of the total decision-making volume into the target layer, the criterion layer and the index layer. The target layer is the health status of the wetland ecosystem in Yunnan Province, the criterion layer is pressure, state, and response, and the index layer includes 15 indicators, such as population density. According to the characteristics of Yunnan wetlands, this study inquired about relevant statistical data, constructed a comparison matrix of influencing factors, and calculated the eigenvalues and eigenvectors of the matrix by MATLAB software. The final result was that the consistency ratio $\mathrm{CR}<0.1$, which means the consistency test is satisfied. Normalize the feature vector to obtain the corresponding weight and its standardized value as shown in Tables 1 and 2.

Fig. 3. Pressure-state-response model framework

Table 1. The health evaluation index system and weight value of Yunnan wetland ecosystem

\begin{tabular}{|c|c|c|c|c|}
\hline \multicolumn{3}{|c|}{$\underline{T}$ Target layer Criterion layer Weights } & Index layer & Weights \\
\hline \multirow{5}{*}{$\begin{array}{l}\text { Health } \\
\text { Status } \\
\text { of }\end{array}$} & \multirow{4}{*}{ pressure(B1) } & \multirow{4}{*}{0.2493} & Population density $(\mathrm{C} 1)$ & 0.3204 \\
\hline & & & Stress degree of wetland(C2) & 0.1394 \\
\hline & & & Water supply(C3) & 0.0855 \\
\hline & & & Wetland area change rate $(\mathrm{C} 4)$ & 0.4547 \\
\hline & \multirow{8}{*}{ status(B2) } & \multirow{8}{*}{0.5936} & Water area $(\mathrm{C} 5)$ & 0.2363 \\
\hline Wetland & & & Flood control(C6) & 0.1265 \\
\hline Ecosystem & & & Wetland water quality $(\mathrm{C} 7)$ & 0.1622 \\
\hline & & & Hydrological regulation $(\mathrm{C} 8)$ & 0.1745 \\
\hline Yunnan & & & Material supply(C9) & 0.0520 \\
\hline Province & & & Scientific research and entertainment $(\mathrm{C} 10)$ & 0.0729 \\
\hline \multirow{5}{*}{ (A) } & & & Habitat protection(C11) & 0.0817 \\
\hline & & & Species diversity(C12) & 0.0938 \\
\hline & \multirow{3}{*}{ response(B3) } & \multirow{3}{*}{0.1571} & Wetland management level(C & 0.4934 \\
\hline & & & Ecological environment restoration(C14) & 0.1958 \\
\hline & & & Wetland construction investment $(\mathrm{C} 15)$ & 0.3108 \\
\hline
\end{tabular}




\begin{tabular}{ccccc}
\hline C9 $(\%)$ & 19.4 & 17.5 & 11.3 & I \\
C10 & 3 & 3 & 3 & III \\
C11(category) & - & 161 & - & II \\
C12(category) & 25434 & 25451 & - & I \\
C13 & 4 & 4 & 4 & II \\
C14 & 3 & 4 & 4 & II \\
C15 & 4 & 4 & 4 & II \\
\hline
\end{tabular}

Note: "-"means the data is not clear.

\subsubsection{Comprehensive evaluation method}

In this paper, a comprehensive evaluation index is used to reflect the health quality level of Yunnan wetland ecosystem. The formula is:

$$
C E I=\sum\left(I_{i} \times W_{i}\right)
$$

In the formula: $C E I$ is the comprehensive evaluation index, $I_{i}$ is the normalized value of a single index, and $W_{i}$ is the normalized weight of the evaluation index.

The comprehensive health index formula of wetland ecosystem pressure, state and response is:

$$
\begin{aligned}
P E I & =\sum\left(I_{i} \times W_{i}\right) / W_{P} \\
S E I & =\sum\left(I_{i} \times W_{i}\right) / W_{s} \\
R E I & =\sum\left(I_{i} \times W_{i}\right) / W_{R}
\end{aligned}
$$

Where PEI, SEI, REI represent the health index of stress, state, and response respectively, $i$ is the number of evaluation indexes in each system. $W_{i}$ is the normalized weight of index $i . I_{i}$ is the normalized value of evaluation index $i . W_{p}, W_{s}$, and $W_{R}$ are the weights of pressure, state, and response indicators.

\section{Result analysis and discussion}

\subsection{Evaluation results}

The weights of the factors at the criterion level of the Yunnan wetland ecosystem health evaluation index system determined by the Analytic Hierarchy Process are pressure 0.2493 , status 0.5936 , and response 0.1571 . The weights of each index layer are shown in Table 1. The comprehensive evaluation index of Yunnan wetland ecosystem obtained by the comprehensive evaluation method is 0.5123 in 2016, 0.5524 in 2017, and 0.3103 in 2018. According to the classification of health evaluation, Yunnan wetland ecosystem belongs to the third level (vulnerable) in 2017, which is close to Level II (sub-health). In 2016 and 2018, some data are not yet clear, so it will not participate in the determination of the level, and only part of the factor analysis will be done. The health index of the stress system is 0.1070 for three consecutive years, and the health index of the state system is 0.2797 in 2017 , and the health index of the response system is 0.1595 in 2016, and 0.1657 in 2017 .

\subsection{Stress analysis}

According to the comprehensive evaluation method, the comprehensive evaluation index of the pressure index is 0.4294 for three years, which is a vulnerable state. In the pressure system, the weights of population density and wetland area change rate to the pressure layer are respectively 0.3204 and 0.4574 . The sum of the weights of the two indicators is greater than 0.5 , which has a greater impact on the stress status of the wetland ecosystem in Yunnan. The impact of water supply and water replenishment is relatively small, and their weights are 0.1394 and 0.0855 respectively.

\subsection{Status analysis}

The comprehensive evaluation index of Yunnan wetland ecosystem status index in 2017 was 0.4711 , which is in a vulnerable state. Among the eight indicators of the state system, water area is an important part of the wetland ecosystem. The weights of water area, hydrological regulation and wetland water quality are $0.2363,0.1745$, and 0.1622 respectively. The sum of the weights of the three indicators is greater than 0.5 . Flood regulation is an important means to protect people's living and property and turns harms into benefits. The weight of the state layer is 0.1265 . Species diversity and habitat protection are the important components of wetlands with weights of 0.0938 and 0.0817 respectively then those are scientific research and entertainment and material supply with the weights to the status layer are 0.0729 and 0.0520 respectively.

\subsection{Response analysis}

The comprehensive evaluation index of the response index was 1 in 2016 and in 2017, which is in a health state. From the perspective of the response system, the level of wetland management is an important factor affecting the healthy development of the wetland ecosystem with a weight of 0.4934 . The investment in wetland construction and ecological environment restoration with weights of 0.3108 and 0.1958 are for the Response layer, respectively.

\subsection{Discussion}

The concept of wetland is currently not unified, but it is a transitional ecosystem between terrestrial ecosystems and aquatic ecosystems. At the same time, there is no relevant standard for the ecosystem health evaluation index system. This paper takes Yunnan as the research example, and the good health status of wetland is representative in the Yangtze River Economic Zone. Using the PSR model as the framework, the wetland ecosystem is regarded as an organic whole, and the health status of the wetland ecosystem in Yunnan is comprehensively analyzed. The combination of remote sensing images improves the accuracy of analysis.

In terms of pressure indicators, it is concluded from the PSR model that the health of the wetland ecosystem 
in Yunnan is mainly affected by the rate of change of wetland area. The wetland area change rate for three consecutive years is less than $1 \%$, because the stability of the wetland area is an important foundation for people's sustainable development each time compared to the previous year's area ratio, followed by human activities that have a certain impact on the degree of stress on the wetland. In this study, the population density of Yunnan averaged 121 people $/ \mathrm{km}^{2}$ in three years. It is in a health state and has a positive effect on wetland construction and ecological benefits. Under the management and protection of government departments, the province has 4 internationally important wetlands and 15 important provincial wetlands.

In terms of status indicators, it is concluded from the PSR model that the health of the wetland ecosystem in Yunnan is mainly affected by the area of water bodies and hydrological regulation. In this study, the three-year average land area of waters and water conservancy facilities in the province is 702,700 hectares, which is fragile and close to sub-health. Wetland plays an important role in water storage, hydrological regulation, replenishment of groundwater and maintaining regional water balance. It is a natural treasure for water storage and flood prevention, and it can distribute uneven precipitation in time and space. From a spatial point of view, lakes and reservoirs in urban areas are greatly affected by pollution. From a time point of view, summer precipitation will increase the water quality of lake areas and reservoirs, and the concentration of pollutants will be reduced. Therefore, relevant departments should increase the protection and reasonable application of water bodies.

In terms of response indicators, it is concluded from the PSR model that the health of the wetland ecosystem in Yunnan is mainly affected by the level of wetland management. In this study, the comprehensive evaluation index of response indicators is in a health status, which is related to the relevant policies of Yunnan Province. With the gradual development of society, people's demand for ecological health is getting higher and higher, and the level of wetland management continues to increase. There were 161 nature reserves of various types and levels at the end of 2017.

The results of this study generally reflect the health status of the wetland ecosystem in Yunnan. However, due to many factors affecting the wetland ecosystem health evaluation indicators, and the current ecological health evaluation indicator system does not have a unified standard, and the final evaluation results are somewhat different. The reasonable selection of the content of the index layer needs further study.

\section{Conclusion}

This study uses remote sensing images and statistical data, and adopts the PSR model framework and the Analytic Hierarchy Process, combining with the comprehensive evaluation method to analyze and evaluate the health of the wetland ecosystem in Yunnan. The main conclusions are as follows:

(1) The comprehensive evaluation index for the health of the wetland ecosystem in Yunnan was 0.5524 in 2017. The health of the wetland ecosystem is at level III (fragile), close to level II (sub-health).

(2) In the three criterion levels of pressure, state, and response, the pressure and state subsystems are in a fragile state, and the response subsystem is in a health status. It shows that the ecological pressure and state system of Yunnan wetlands have relative risks, and the response indicators should continue to maintain the healthy state of the ecosystem.

(3) In the wetland ecosystem health evaluation index system of Yunnan, wetland area change rate, water body area, hydrological adjustment and wetland management level are the most important factors affecting the health of Yunnan wetland ecosystem.

This work is jointly supported by Drought Meteorological Science Research Foundation (IAM201902) and Research on quality detection method of land cover classification based on depth confidence neural network (201901).

\section{References}

1. Y.X. Yang, Main Characteristics, Progress and Prospect of International Wetland Science Research. PIG, 21(2002)

2. H.J. He, X.G. Liu, L. Han, L.Chen, The assessment of wetland ecosystem based on RS image. ESM, 20(2011)

3. X.Q. Mao, Ecological and Environmental Health Assessment of Wetland in Dongting Lake. SE, 39(2020)

4. F.Q. Wang, P.H. Liu, H. Yang, H. Zhao, PSR model-based evaluation on ecosystem health of tail-reach wetland of Diaokouhe River. WRHE, 50(2019)

5. Y. Xu, F. Yang, C.Z. Yan, Ecological health assessment of urban wetland in Xiong'an based on landscape pattern. AES, 40(2020)

6. Y.Q. Shu, W.F. Song, Research progress of wetland ecosystem health assessment in my country. SSWC, 32(2020)

7. C. Ma, Remote Sensing and landscape analysis of Wetlands in Northeast China. JH, 38(2017)

8. C.J. Zhu, B. Tian, Y.X. Zhou, Z.Q. Fan, Wetland Change Analysis and Forecasting in Pudong New Area Using Markov and CLUE-S Model. JFU,Natural Science,54(2015)

9. A.N. Suo, D.Z. Zhao, B.Q. Wei, Y.L. Chen, Value evaluation on ecosystem function of wetland in Liaohe River Delta. MES, 28(2009)

10. L.C. Wang, L. Jiao, F.B. Lai, N.M. Zhang, Evaluation of ecological changes based on a remote sensing ecological index in a Manas Lake wetland, Xinjiang. AES, 39(2019)

11. S.W. Bian, Simple Discussion on Distribution and Characteristics of Yunnan Wetland Type. FIP, 31(2006) 